\title{
Nonsingular Terminal Sliding Mode Control for the Speed Regulation of Permanent Magnet Synchronous Motor with Parameter Uncertainties
}

\author{
Wei $\mathrm{Xu}^{1}$, Senior Member, IEEE, Yajie Jiang ${ }^{1}$, Chaoxu $\mathrm{Mu}^{2}$, Member, IEEE and Hong Yue ${ }^{3}$, Senior Member, IEEE \\ ${ }^{1}$ State Key Laboratory of Advanced Electromagnetic Engineering and Technology, \\ School of Electrical and Electronic Engineering, Huazhong University of Science and Technology, Wuhan, China \\ ${ }^{2}$ School of Electrical and Automation Engineering, Tianjin University, Tianjin, China \\ ${ }^{3}$ Department of Electronic and Electrical Engineering, University of Strathclyde Glasgow, Scotland, UK \\ E-mail: weixu@hust.edu.cn
}

\begin{abstract}
The drive performance of permanent magnet synchronous motor (PMSM) can be deteriorated due to various disturbances. In this paper, the problem of speed control for a PMSM system with parameter uncertainties is investigated. A new control algorithm based on nonsingular terminal sliding mode control (NTSMC) is proposed, where the controller is developed for speed regulation. Compared with conventional strategies, this new controller provides improved performance for speed regulation of PMSM when subject to parameter uncertainties, in that it achieves fast dynamic response and strong robustness. Simulation studies are conducted to verify the effectiveness of this proposed method.
\end{abstract}

\section{INTRODUCTION}

Permanent magnet synchronous motors (PMSM) are widely used in industry owing to superior features such as compact structure, high efficiency, high torque to inertia ratio, and high power density, , particularly in applications of electric vehicles, airplanes, electrical-drive ships and so on [1-4].

Despite of all these advantages, the use of PMSMs is often subject to various disturbances [5], which may be caused by either internal or external factors, for instance, friction force, working temperature and load disturbances. These disturbances can be characterized by parametric uncertainties in a PMSM model [6], which may deteriorate the performance of a PMSM system, such as a decreased estimation accuracy of the rotor position. Meanwhile, PMSM is a typical highly nonlinear and multivariable system [7], and its performance is sensitive to parameter variations.

A number of useful control strategies have been developed for improved operation of PMSM servo systems from different aspects, such as sliding mode control (SMC) [8], proportion-integral-derivative (PID) control [9], active disturbance rejection control [10], adaptive control [11], field-oriented control [12], chaos control [13], predictive control [14], back stepping control[15], nonlinear control [16] and so on Among them SMC is a robust method against disturbances and parameter uncertainties [17], however, there are certain disadvantages in applying SMC to PMSMs, : one is the inherent chattering with the discontinuous control variable; the other is that SMC normally produces a large gain to restrain disturbances. The boundary layer is introduced to overcome chattering $[18,19]$, but it is difficult to completely remove chattering
[20], especially when the width of boundary layer is very small or the control gain is very large [21]. Therefore SMC cannot guarantee the robustness in the presence of parameter uncertainties. Alternative methods need to be explored to increase the PMSM robustness ability. This motivates our work in this paper.

Nonsingular terminal sliding mode control (NTSMC) is known to be insensitive to parameter variations and disturbances. NTSMC not only has advantages in fast dynamic response, finite time convergence, high control precision [8], but can also eliminate the paranormal phenomenon in the control input of the system. Compared with the traditional sliding mode control, NTSMC method is more capable of reducing chattering.

Considering the PMSM drive performance, the vector control scheme and the cascade control structure is adopted [21, 22] in NTSMC in this paper. Under this scheme, the flux producing components of the stator current and the torque are decoupled so that the independent torque and flux controls are possible as in DC motors. The cascade architecture offers advantages including the ability to resist parameter uncertainties and the improved response performance. Furthermore, one speed loop and two current loops composite the vector control scheme. A random parameter generator is added in the PMSM speed regulation system, which produces random parameters to cope with parameter uncertainties. Then an NTSM controller is designed to achieve the desired tracking performance in the PMSM speed system.

\section{PMSM MODEL DESCRIPTION}

A general PMSM model in the $d-q$ reference coordinates can be described as follows $[5,6]$

$$
\left[\begin{array}{c}
\dot{i_{d}} \\
\dot{i_{q}} \\
\dot{\omega}
\end{array}\right]=\left[\begin{array}{ccc}
-\frac{R_{s}}{L_{d}} & n_{p} \omega & 0 \\
-n_{p} \omega & -\frac{R_{s}}{L_{q}} & -\frac{n_{p} \psi_{f}}{L_{q}} \\
0 & \frac{1.5 n_{p} \psi_{f}}{J} & -\frac{B}{J}
\end{array}\right]\left[\begin{array}{l}
i_{d} \\
i_{q} \\
\omega
\end{array}\right]+\left[\begin{array}{l}
\frac{u_{d}}{L_{d}} \\
\frac{u_{q}}{L_{q}} \\
-\frac{T_{l}}{J}
\end{array}\right]
$$

where $i_{d}$ and $i_{q}$ are $d$ - and $q$-axis stator currents respectively, $L_{d}$ and $L_{q}$ are $d$ - and $q$-axis stator inductances respectively, $u_{d}$ and $u_{q}$ are $d$-and $q$-axis stator 
voltages respectively, $R_{s}$ is the stator resistance, $\omega$ is the rotor angular velocity, $n_{p}$ is the number of pole pairs, $\psi_{f}$ is the flux linkage, $T_{l}$ is the load torque, $B$ is the viscous friction coefficient, and $J$ represents the moment of inertia.

The PMSM model in (1) is clearly nonlinear and coupled in view of the product of the angular velocity $\omega$ and the stator currents $i_{d}$ and $i_{q}$. The vector control strategy is well known since it can decouple the PMSM states, where a cascade control structure is adopted that includes a speed loop and two current loops. Usually, the $\mathrm{d}$-axis reference current $i_{d}^{*}$, is set as $i_{d}^{*}=0$ in order to approximately eliminate the coupling between the angular velocity and the stator currents. In other words, $\omega$ and $i_{q}$ are decoupled when $i_{d}=i_{d}^{*}=0$. Fig. 1 shows the control structure of the PMSM system based on vector control.

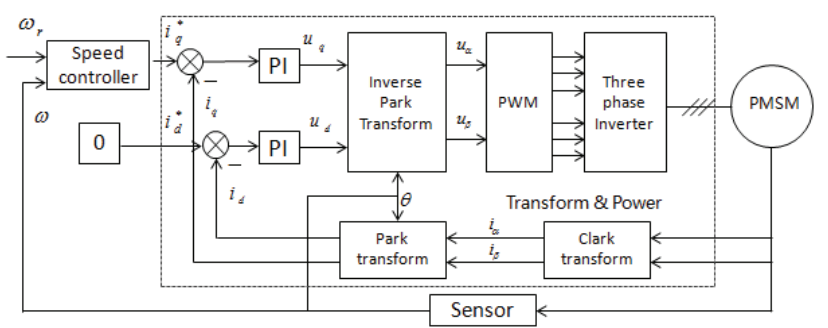

Fig. 1. The decoupling control diagram of PMSM system based on vector control.

From model (1), the relationship between the angular velocity and $q$-axis current is presented as follows,

$$
\dot{\omega}=\frac{1.5 n_{p} \psi_{f}}{J} i_{q}-\frac{B}{J} \omega-\frac{T_{l}}{J}
$$

With the Laplace transformation, it can be further rewritten as:

$$
s \dot{\omega}(s)=\frac{1.5 n_{p} \psi_{f}}{J} \tilde{i}_{q}(s)-\frac{B}{J} \tilde{\omega}(s)-\frac{T_{l}}{J}
$$

where $\tilde{\omega}(s)$ and $\tilde{i}_{q}(s)$ are the Laplace transformation of $\omega$ and $i_{q} . i_{q}$ can be expressed by the reference current $i_{q}^{*}$ and the $q$-axis voltage $u_{q}$, i.e.,

$$
\tilde{i}_{q}(s)=\tilde{i}_{q}^{*}(s)-\frac{\tilde{u}_{q}(s)}{k_{p}+k_{i} / s}
$$

where $k_{p}$ and $k_{i}$ are the proportional and integral gains of the PI controller in the current loop for $i_{q} \cdot \tilde{i}_{q}^{*}(s)$ and $\tilde{u}_{q}(s)$ are the Laplace transformation of the reference current $i_{q}^{*}$ and the $q$-axis voltage $u_{q}$. Let $k_{f}=1.5 n_{p} \psi_{f}$, the angular velocity $\omega$ in (2) can be changed to link with $i_{q}^{*}$, as represented by

$$
\begin{aligned}
\left(s+\frac{k_{i}}{k_{p} s}\right) \tilde{\omega}(s)=\frac{k_{f}}{J}\left(1+\frac{k_{i}}{k_{p} s}\right) \tilde{i}_{q}^{*}(s)- & \frac{k_{f}}{k_{p} J} \tilde{u}_{q}(s) \\
& -\frac{B}{k_{p} J} \tilde{\omega}(s)-\frac{T_{l}}{k_{p} J}
\end{aligned}
$$

Considering a second order angular velocity model, let the controller and the reference current $i_{q}$ have the following relationship

$$
\frac{1}{s} \tilde{U}(s)=\frac{k_{f}}{J}\left(1+\frac{k_{i}}{k_{p} s}\right) \tilde{i}_{q}^{*}(s)
$$

Therefore the second-order angular velocity model is obtained to be

$$
\ddot{\omega}=U-\frac{B k_{p}+k_{i} J}{k_{p} J} \dot{\omega}-\frac{k_{i} B}{k_{p} J} \omega-\frac{k_{f}}{k_{p} J} u_{q}-\frac{\dot{T}_{l}}{k_{p} J}-\frac{k_{i} T_{l}}{k_{p} J}
$$

Denoting $f(t)=\frac{B k_{p}+k_{i} J}{k_{p} J} \dot{\omega}+\frac{k_{i} B}{k_{p} J} \omega, d(t)=\frac{k_{f}}{k_{p} J} u_{q}+\frac{\dot{T}_{l}}{k_{p} J}+\frac{k_{i} T_{l}}{k_{p} J}$ to represent lumped uncertainty term, equation (7) is rewritten as

$$
\ddot{\omega}=U-f(t)-d(t)
$$

The control objective is to design a nonsingular terminal sliding mode controller to regulate the PMSM angular velocity.

\section{Nonsingular Terminal SLiding Mode Control FOR THE PMSM SPEED REGULATION}

\section{A. Design of Nonsingular Terminal Sliding Mode Strategy}

A second-order nonlinear dynamic system with uncertainties is considered as follows,

$$
\left\{\begin{array}{l}
\dot{x}_{1}(t)=x_{2}(t) \\
\dot{x}_{2}(t)=f(x(t), t)+u(t)+d(t)
\end{array}\right.
$$

where $f(\boldsymbol{x}(t), t)$ is an unknown function, the estimated value of $f(\boldsymbol{x}(t), t)$ is denoted as $\hat{f}(\boldsymbol{x}, t)$, which is a simplified function that is closed to $f(x(t), t)$. $d(t) \leq D(t)$ is taken as a bounded disturbance signal. The following constraint is satisfied:

$$
\Delta f(x(t), t)=f(x(t), t)-\hat{f}(x(t), t) \leq F(x(t), t)
$$

where $F(\boldsymbol{x}(t), t)$ is a known function. A new sliding mode variable is designed according to the theory of NTSMC,

$$
s=x_{1}+\frac{1}{\beta} x_{2}^{p / q}
$$


where $\beta>0$ is constant, $p$ and $q$ are odd number, $0<q<p$. Combining with (9), the sliding control law is designed as follows:

$$
\dot{s}=\dot{x}_{1}+\frac{1}{\beta} \frac{p}{q} \dot{x}_{2} x_{2}^{p / q}+(F(\boldsymbol{x}, t)+D(t)+\eta) \operatorname{sgn}(s)
$$

According to the following analysis, these conditions can guarantee the finite time convergence of the stability of the system.

Taking $u_{e q}(\boldsymbol{x}, t)$ as the equivalent control law derived from setting $\dot{s}=0$, which ensures the error variable $e$ staying on the sliding mode surface $s=0$. Then the equivalent control law is written as

$$
u_{e q}(\boldsymbol{x}, t)=\hat{f}(\boldsymbol{x}, t)+\ddot{x}_{d}+\beta \frac{q}{p} \dot{e}^{2-q / p}
$$

The control law design of NTSMC is

$$
u(t)=u_{e q}(\boldsymbol{x}, t)+u_{s w}(\boldsymbol{x}, t)
$$

$k(\boldsymbol{x}, t)$ is the nonlinear gain as depicted by

$$
k(\boldsymbol{x}, t)=F(\boldsymbol{x}, t)+D(t)+\eta
$$

In order to robustly drive the sliding variables converge to zero in finite time and considering the $d(t)$, second order super twisting algorithm is used to design the switching control

$$
\dot{s}=-k(\boldsymbol{x}, t) \operatorname{sgn}(s(t))
$$

From (13) to (16), the following controller can be developed

$$
u(t)=\hat{f}(\boldsymbol{x}, t)+\ddot{x}_{d}+\beta \frac{q}{p} \dot{e}^{2-q / p}+(F(\boldsymbol{x}, t)+D(t)+\eta) \operatorname{sgn}(s(t))
$$

\section{B. Nonsingular Terminal Sliding Mode Control For PMSM}

The parameter uncertainties of the PMSM speed regulation system are described as $d(t)$ in this paper. In the system (7), the desired rotor speed is denoted as $\omega_{r}$, and the tracking error is $e=\omega_{r}-\omega$ for the speed regulation system of PMSM.

Combining the PMSM equations (8) and (9), it will get

$$
\left\{\begin{array}{l}
\dot{x}_{1}=x_{2}=\dot{\omega}_{r}-\dot{\omega} \\
\dot{x}_{2}=\ddot{\omega}_{r}-U+f(t)+d(t)
\end{array}\right.
$$

where $\eta>0$ is a constant.

The sliding mode variable $s$ is designed by (11) and (18), and then it will get

$$
s=\omega_{r}-\omega+\frac{1}{\beta}\left(\dot{\omega}_{r}-\dot{\omega}\right)^{p / q}
$$

Take time derivative of the sliding mode variable $s$, which is

$$
\dot{s}=\dot{\omega}_{r}-\dot{\omega}+\frac{1}{\beta} \frac{p}{q}\left(\ddot{\omega}_{r}-U+f(t)+d(t)\right)\left(\dot{\omega}_{r}-\dot{\omega}\right)^{1-p / q}
$$

From (9) and (10), it can be derived to have

$$
\ddot{\omega}_{r}-U+f(x)+d(t) \leq \ddot{\omega}_{r}-U+F(\boldsymbol{x}, t)+D(t)
$$

Thus the second-order NTSMC becomes

$$
U=U_{e q}+U_{s w}
$$

Combining (17) - (19), the following control strategy is obtained:

$$
\begin{aligned}
U=\ddot{\omega}_{r}+f(t)+\beta \frac{q}{p}\left(\dot{\omega}_{r}-\dot{\omega}\right)^{2-p / q} & \\
& +(F(\boldsymbol{x}, t)+D(t)+\eta) \operatorname{sgn}(s)
\end{aligned}
$$

In the simulation experiments, an equivalent reduction can be made:

$$
U=\ddot{\omega}_{r}+f(t)+\beta \frac{q}{p}\left(\dot{\omega}_{r}-\dot{\omega}\right)^{2-p / q}+k \operatorname{sgn}(s)
$$

where $k>0$ is a constant. For further simplification, there is

$$
\begin{aligned}
U=\ddot{\omega}_{r}+\frac{k_{i}}{k_{p}} \dot{\omega}+\beta \frac{q}{p}\left(\dot{\omega}_{r}\right. & -\dot{\omega})^{2-p / q} \\
& +k \operatorname{sat}\left(\omega_{r}-\omega+\frac{\left(\dot{\omega}_{r}-\dot{\omega}\right)^{p / q}}{\beta}\right)
\end{aligned}
$$

Combining (25) with (19), there is

$$
U=\ddot{\omega}_{r}+\dot{\omega} \frac{k_{i}}{k_{p}}+\beta \frac{q}{p}\left(\dot{\omega}_{r}-\dot{\omega}\right)^{2-p / q}+\operatorname{sat}(s) k
$$

In sum, with the controller (26), the proof is thus completed.

According to the control diagram in Fig.1, the real output of the speed controller is the $q$-axis reference current $i_{q}^{*}(t)$. With the relationship between $U(t)$ and $i_{q}^{*}(t)$ expressed in (6), the signal of $i_{q}^{*}(t)$ is obtained by

$$
\tilde{i}_{q}^{*}(s)=\frac{k_{p} J}{k_{f}\left(k_{p} s+k_{i}\right)} \tilde{U}(s)
$$

The nonsingular terminal sliding mode control for the PMSM speed regulation is shown in Fig. 2.

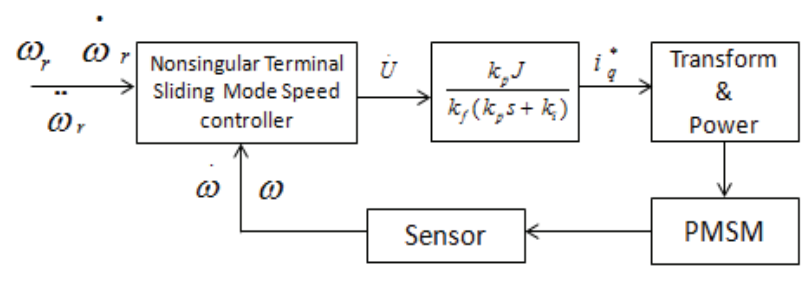

Fig.2. The relationship of $\tilde{U}$ and $\tilde{i}_{q}^{*}$ in the PMSM system.

In order to make simulation study to real application of PMSM as close as possible, a Random Parameter module is added to the control scheme. This module produces 
random parameters, which are regarded as the parameter uncertainties. The completed NTSMC scheme for PMSM speed regulation is presented in Fig. 3.

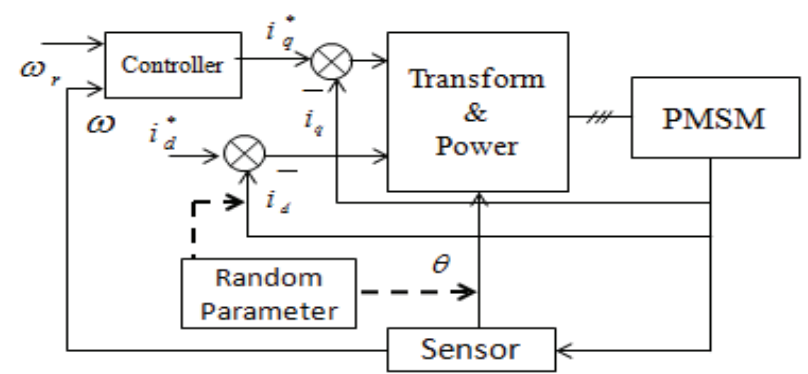

Fig.3. Speed relationship of PMSM system with parameter uncertainties based on NTSMC.

\section{Simulation ANALYsis}

In order to examine the proposed control strategy, a PMSM speed control system is built in MATLAB. Simulation for the proposed NTSMC strategy is performed on a PMSM with the parameters listed in Table I.

TABLE I. PARAMETERS OF THE PMSM

\begin{tabular}{ll}
\hline Number of phases & 3 \\
Number of poles & 4 \\
Armature resistance & $1.74 \Omega$ \\
Rated power & $750 \mathrm{~W}$ \\
Rotor flux linkage & $0.402 \mathrm{wb}$ \\
Stator inductance & $0.004 \mathrm{H}$ \\
Viscous damping & $7.403 * 10^{-5} \mathrm{~N} \cdot \mathrm{m} \cdot \mathrm{s} / \mathrm{rad}$ \\
Moment of inertia & $1.78 * 10^{-4} \mathrm{~kg} \cdot \mathrm{m}^{2}$ \\
\hline
\end{tabular}

The simulation period is 0.1 second, and the torque $T_{l}$ $=4 \mathrm{Nm}$ from $t=0 \mathrm{~s}$ and is remained at this level, the Random Parameter module produces random parameters, which are regarded as the parameter uncertainties.

\section{A. Parameter Uncertainties Added at Rotor Position Loop}

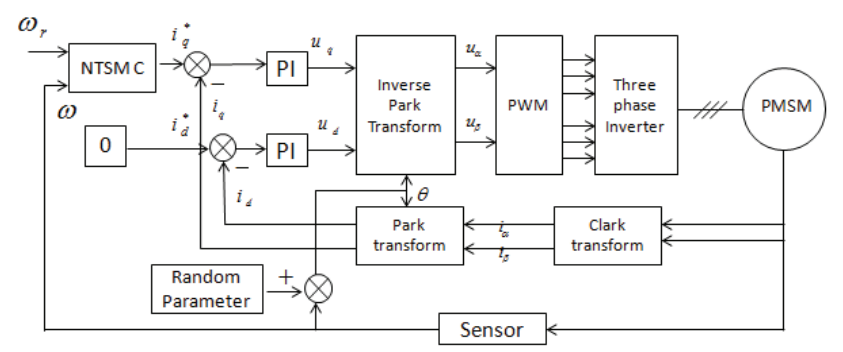

Fig.4. The control scheme for the PMSM system with parameter uncertainties added at position feedback loop.

As shown in the Fig.4, the random parameter is added to the feedback loop, which are bounded from -0.2 to $+0.2 \mathrm{rad}$ per 0.01 second. The reference angular velocity of
PMSM is set to be $\omega_{r}=150 \mathrm{rad} / \mathrm{second}$. Simulation results are shown in Figs. 5 and 6, respectively.

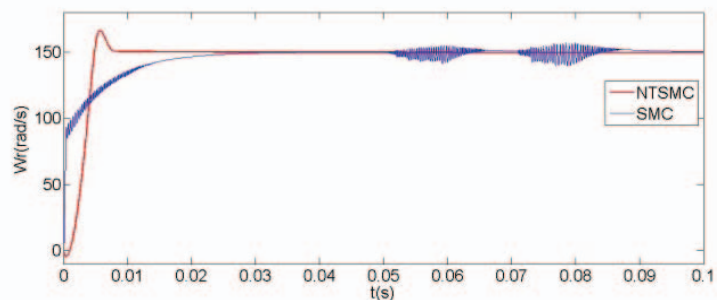

(a)

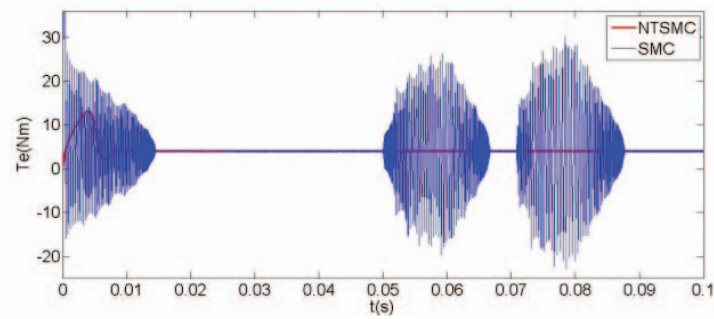

(b)

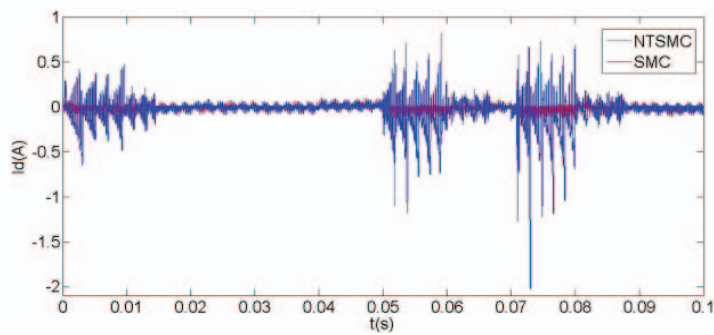

(c)

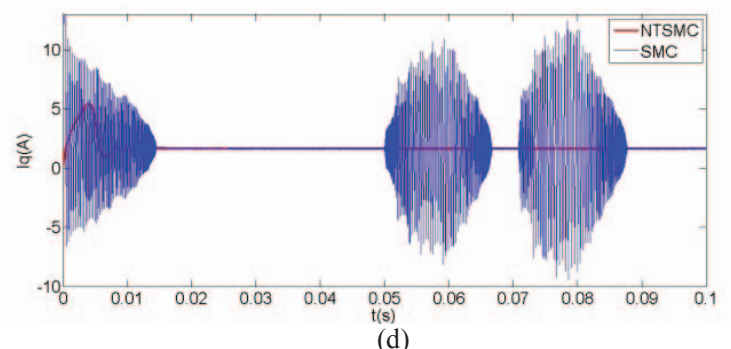

Fig.5. $\omega, T_{e}, i_{d}$ and $i_{q}$ in the PMSM system with parameter uncertainties. (a) The speed regulation of PMSM system. (b) The motor torque. (c) The d axis stator current. (d) The q axis stator current.

Tracking dynamic performances of PMSM system based on the general SMC and the proposed NTSMC were compared in (a) - (d) subfigures in Fig.5, respectively, the red curve describes the control performance based on the proposed NTSMC, and the blue curve illustrates the control performance based on SMC. Fig.5 (a) describes the speed control performance with the proposed speed controller. When the torque is introduced, the response of the PMSM system under NTSMC is faster than that under SMC. In addition, the chattering of the PMSM system with $\mathrm{SMC}$ is more serious, the torque error under $\mathrm{SMC}$ is also larger. Compared with SMC, NTSMC can track the given 
speed quicker with a stronger disturbance rejection performance.

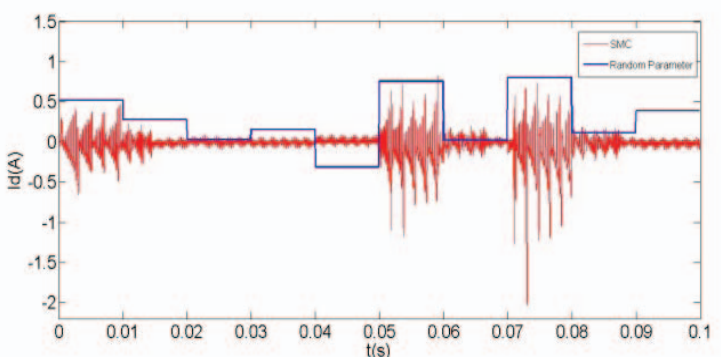

(a)

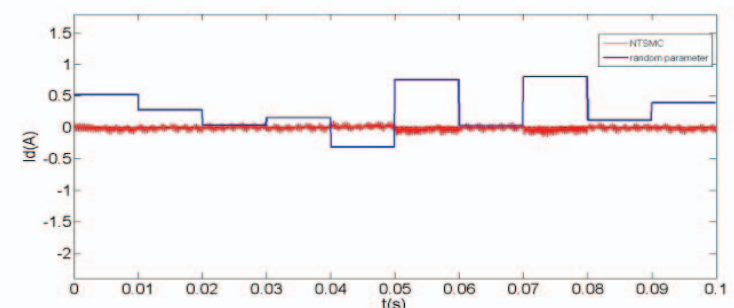

(b)

Fig.6. The comparison of the random parameters and $i_{d}$.

As shown in Fig.6, the red curve describes the $d$-axis stator current control performance with SMC (a) and NTSMC (b), and the blue curve describes the parameter uncertainties. When the uncertain parameters are changing, the chattering of SMC is very severe, the range is hence very large. By contrast, NTSMC shows a much better control performance: the chattering is very small; there are no obvious changes following the increase or decrease of the random parameter variations. By comparing the results of NTSMC and SMC, it can the concluded that the robustness of PMSM speed regulation is enhanced with the proposed NTSMC.

\section{B. Parameter Uncertainties Added at d-Axis Stator Current Loop}

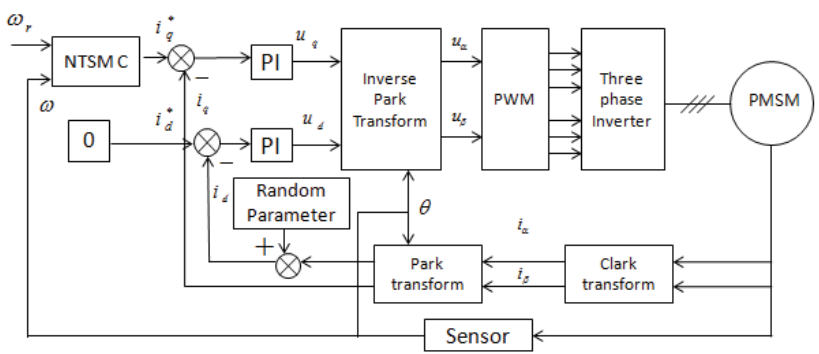

Fig.7. The control scheme for the PMSM system with parameter uncertainties added at $d$-axis current loop.

As shown in the Fig.7, the random parameter is added at $d$-axis current loop, which are bounded from -11.9 to $+11.9 \mathrm{~A}$ per 0.01 second. The rotor reference angular velocity of PMSM is $150 \mathrm{rad} / \mathrm{second}$ too. Simulation results are shown in Figs. 8 and 9, respectively.

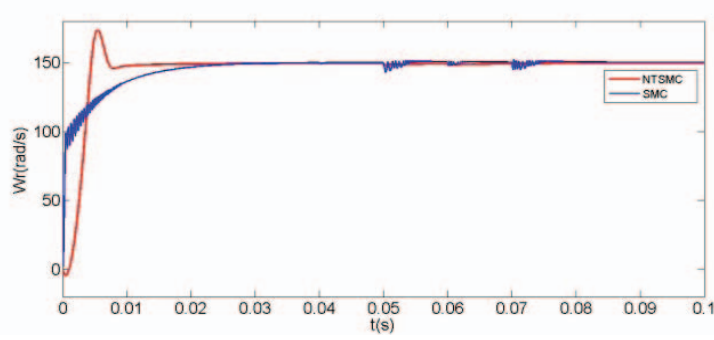

(a)

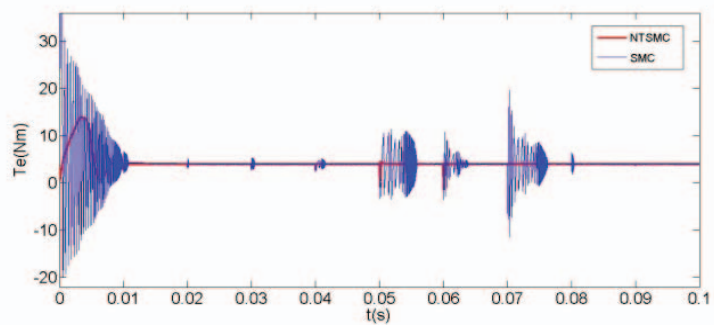

(b)

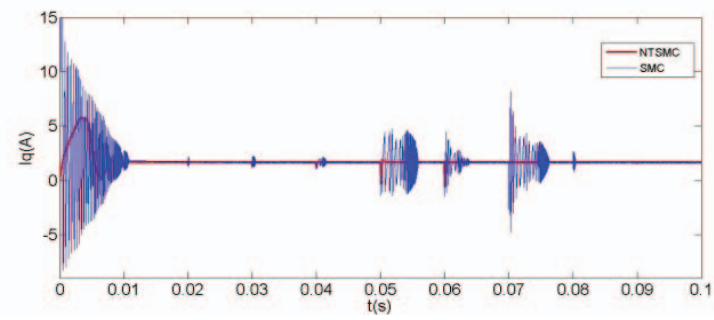

(c)

Fig. 8. $\omega, T_{e}$ and $i_{q}$ in the PMSM system with parameter uncertainties. (a) The speed regulation of PMSM system. (b) The motor torque with parameter uncertainties. (c) $q$-axis stator current.

As shown in Fig. 8, the blue curve describes the control performance under the SMC, and the red curve describes the control performance under the proposed NTSMC. When the random parameters are included, compared with SMC, is the NTSMC has a faster time response and a smaller tracking error. In addition, the chattering of the PMSM system is largely reduced with the NTSMC approach.

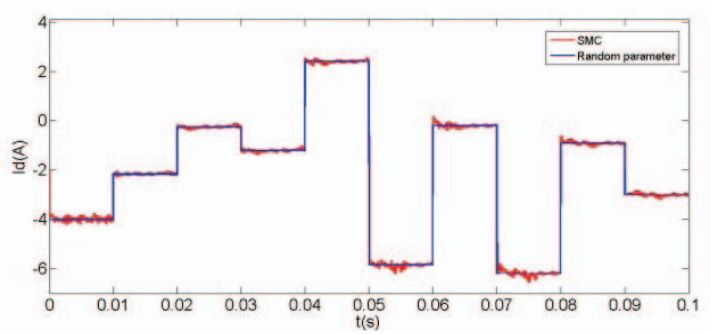

(a) 


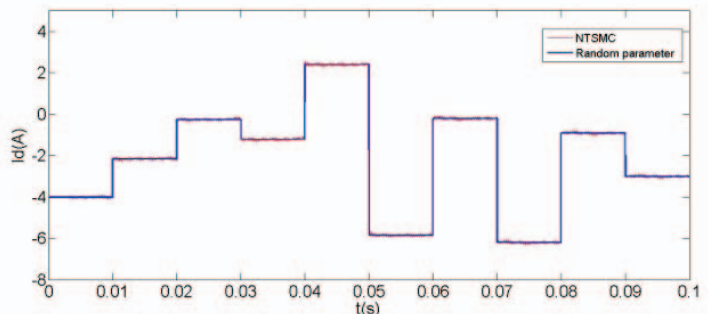

(b)

Fig. 9. The comparison of the opposite of random parameters and $d$-axis stator current

In view of the $d$-axis current loop is a negative feedback loop, and the $d$-axis reference current is $0 \mathrm{~A}$, the random parameters are added at $\mathrm{d}$ axis stator current loop simultaneously. So it takes the opposite of random parameters and comparing with the $d$-axis current respectively as shown in Fig.9, in which the red curve describes the d-axis stator current control performance with the proposed SMC (a) and NTSMC (b), and the blue curve describes the opposite of parameter uncertainties. It can be observed that the proposed two methods can both track the parameter uncertainties. The chattering has been reduced greatly based on nonsingular terminal sliding mode control, and two curves almost perfect coincidence. Hence, by careful investigation and analysis, it is known the NTSMC method has better robustness ability when the parameter changes.

\section{CONCLUSION}

A new NTSMC strategy has been proposed for the speed regulation of a PMSM system under parameters uncertainties. The simulation study shows that the proposed NTSMC can achieve satisfactory PMSM speed regulation performance with random parameter uncertainties. However, when the disturbances are at a more serious level, the chattering effect under NTSMC will be difficult to handle using the current strategy. Further study is undertaking to tackle this problem.

\section{ACKNOWLEDGMENT}

This work was supported by National Natural Science Foundation of China (Nos. 61304018, 51377065, 61301035 and 61411130160), Hubei Province Science and Technology Supporting Program (2014BAA035), Tianjin Natural Science Foundation (14JCQNJC05400), China Post-doctoral Science Foundation (2014M561559), Research Innovation Program of Huazhong University of Science and Technology (2014TS149) and Research Innovation Program of Tianjin University (2013XQ0101).

\section{REFERENCES}

[1] Y. Zhang, C. M. Akujuobi, W. H. Ali, C. L. Tolliver and L.-S. Shieh, "Load disturbance resistance speed controller design for PMSM," IEEE Trans. Ind. Electron, vol. 53, no. 4, pp. 1198-1208, Jun 2006.
[2] T. Ishikawa, Y. Seki and N. Kurita, "Analysis for fault detection of vector-controlled permanent magnet synchronous motor with permanent magnet defect," IEEE Trans. Magn, vol. 49 , no. 5, pp. 2331 - 2334, May 2013.

[3] Y. Xia, X. Yu, and W. Oghanna, "Adaptive robust fast control for induction motors," IEEE Trans. Ind. Electron, vol. 47, no. 4, pp. 854-862, Aug 2000.

[4] C. Kim, T. Kim and J. Lyou, "Robust speed control of a PMSM through sliding mode controller design", in Proc. IEEE Symposium on Industrial Electronics and Applications (ISIEA), Sep 2011, pp. 30-35.

[5] C. Mu, W. Xu and X. Yu. "A continuous sliding mode controller for the PMSM speed regulation based on disturbance observer," in Proc. Conf. of IEEE Industrial Electronics Society (IECON), Oct 2014, pp. 28-33.

[6] S. Li, Z. Liu, "Adaptive speed control for permanent-magnet synchronous motor system with variations of load inertia," IEEE Trans. Ind. Electron, vol. 56, no. 8, pp. 3050-3059, Jun 2009.

[7] L. H, Z. M and Y. H. "Disturbance observer based terminal sliding mode control method for PMSM speed regulation system," in proc. Chinese Control Conf (CCC), Jul 2011, pp. 6287-6292.

[8] F. Lin, P. Chou, C. Chen and Y. Lin, "Three-degree-of-freedom dynamic model-Based intelligent nonsingular terminal sliding mode control for a gantry position Stage," IEEE Trans. Fuzzy Syst, vol. 20, no. 5, pp. 971-985, Mar 2012.

[9] J. Jung, V. Leu, T. Do, E. Kim and H. Choi, "Adaptive PID speed control design for permanent magnet synchronous motor drives," IEEE Trans. Power Electron, vol. 30, no. 2, pp. 900-908, Mar 2015.

[10] S. Hebertt, L. Jesús, G. Carlos and A. C. Marco, "On the control of the permanent magnet synchronous motor: an active disturbance rejection control approach,", IEEE Trans. Control Syst. Technol, vol. 22, no. 5, pp.2056-2063, Jan 2014.

[11] F. Lin, P. Shen, "Adaptive fuzzy-neural-network control for a DSPbased permanent magnet linear synchronous motorservo drive," IEEE Trans, Fuzzy Syst, , vol: 14, no. 4, Page(s): 481 - 495, Aug 2006.

[12] K. Gulez, A. A. Adam and H. Pastaci, "Torque ripple and EMI noise minimization in PMSM using active filter topology and fieldoriented control," IEEE Trans. Ind. Electron, vol. 55 , no. 1, pp. 251-257, Jan 2008.

[13] Z. Wang, K. T. Chau and L. Jian, "Chaoization of permanent magnet synchronous motors using stator flux regulation," IEEE Trans. Magn, vol. 44, no. 11, pp. 4151-4154, Nov 2008.

[14] M. Preindl, S. Bolognani, "Model predictive direct speed control with finite control set of PMSM drive systems,", IEEE Trans. Power Electron, vol. 28 , no. 2, pp. 1007-1015, Jun 2013.

[15] M. Morawiec, "The adaptive backstepping control of permanent magnet synchronous motor supplied by current source inverter,' IEEE Trans. Ind. Informat, vol. 9, no. 2 , pp. 1047 -1055, Oct 2013.

[16] H. Ren ; D. Liu, "Nonlinear feedback control of chaos in permanent magnet synchronous motor," Circuits and Systems II: Express Briefs, IEEE Trans, vol. 53, no. 1, pp. 45-50, Jan 2006.

[17] R. Wai, "Total sliding-mode controller for PM synchronous servo motor drive using recurrent fuzzy neural network," IEEE Trans. Ind. Electron, vol. 48, no. 5, pp. 926 -944, Oct 2001.

[18] I. Baik, K. Kim and M. Youn, "Robust nonlinear speed control of PM synchronous motor using boundary layer integral sliding mode control technique," IEEE Trans. Control Syst. Technol, vol.8, no.1, pp. 47-54. Jan 2000.

[19] H. Kim, J. Son and J. Lee, "A high-Speed sliding-mode observer for the sensorless speed control of a PMSM," IEEE Trans. Ind. Electron, vol. 58, no. 9, pp. 4069-4077, Dec 2011.

[20] Y. Feng, J. Zheng, X. Yu and N. V. Truong, "Hybrid terminal sliding-mode observer design method for a permanent-magnet synchronous motor control system," IEEE Trans Ind. Electron. vol. 56, no.9, pp. 3424-3431, Jun 2009.

[21] S. Li, M. Zhou and X. Yu, "Design and implementation of terminal sliding mode control method for PMSM speed regulation system," IEEE Trans. Ind. Informt, vol. 9, no. 4, pp. 1879-1891, Nov 2013.

[22] M. Rahman, M. Hoque "On-line adaptive artificial neural network based vector control of permanent magnet synchronous motors," IEEE Trans. Energy Convers, vol. 13, no. 4, pp. 311-318, Dec 1998. 